\title{
Blood utilization and quality indicators - trend at a superspecialty hospital in Northern India
}

\begin{abstract}
Blood components are a scarce and expensive resource. Therefore, there is a need to continuously monitor blood utilization and audit the transfusion practices to identify key areas of concern for blood usage. We assessed the transfusion data for the patients requiring blood transfusion at our hospital for a period of one year from January 2015 to December 2015 using patient transfusion requisition forms, crossmatch and issue records. A total of 2434 blood transfusion requisitions were received by the Department of Transfusion Medicine for 3621 (49.69\%) packed red cells (PRBC) units, 2140(27.59\%) fresh frozen plasma (FFP), 1923(24.80\%) random donor platelet concentrates (RDP), 15(0.19\%)whole blood units and 55(0.56\%) single donor apheresis platelet concentrates (SDP). The overall average CT Ratio (1.57), Transfusion probability $(79.0 \%)$ and Transfusion index (1.18) demonstrated that there is efficient blood utilization at our hospital.
\end{abstract}

Keywords: blood components, transfusion hemoglobin, surgical specialities
Volume 2 Issue 3 - 2016

\section{Daljit Kaur, Manoj Kandwal}

Daljit Kaur, Manoj Kandwal

Correspondence: Daljit Kaur, Consultant, Department of Transfusion Medicine, Max Super speciality Hospital, Dehradun, India, 24800।,Tel 9I 88267889II, Fax 01356673555 , Email doc.daljit@gmail.com, drdaljit@outlook.com

Received: March 10, 2016 | Published: May 23, 2016
Abbreviations: PRBC, packed red cells; FFP, fresh frozen plasma; RDP, random donor platelet; SDP, single donor apheresis platelet; TI, transfusion index; MSBOS, maximum surgical blood ordering schedule; CTVS, cardiothoracic and vascular surgery; NSU, neurosurgery unit

\section{Introduction}

Blood being a biologic agent, is associated with both infectious and noninfectious complications. As blood components are a scarce and expensive resource, inappropriate blood transfusions need to be avoided. Therefore, there is a need for continuous monitoring of blood utilization and auditing the transfusion practices which serves in identifying key areas of concern in the blood component usage as well as instances of inappropriate component use, wherein corrective actions can be planned. Ours is a 200 bedded hospital with average bed occupancy of 120 and there is self sufficiency for provision of blood components to the admitted patients. This study was designed to analyze the trend of utilization of different blood components by various medical and surgical specialities at our hospital by determining the quality indicators for the same. The various quality indicators for blood utilization so studied were calculated as:

Crossmatch to transfusion ratio $($ CT ratio) $=$ number of units crossmatched/number of units transfused

Transfusion Probability $(\% \mathbf{\%})=$ number of patients transfused/ number of patients crossmatchedx 100

Transfusion index (TI )=number of units transfused/number of patients transfused

\section{Maximum surgical blood ordering schedule $($ MSBOS $)=1.5 \times \mathrm{xI}$.}

\section{Methodology}

i. Study Period: January 2015 to December 2015 (one year), the data for quality indicators was analyzed from a representative first half of the year (January2015 to June 2015) simulating the entire year's issue of blood components. ii. Study design: Cross-sectional prospective study.

iii. Inclusions: All the patients requiring blood component transfusion for various specialities of our hospital.

iv. Modalities: Patient transfusion request forms, crossmatch and issue records.

v. Data Inclusions: Age, gender, blood group, consultant in charge, specialty where the patient was admitted, diagnosis and indication of transfusion, pre-transfusion hemoglobin/ platelet count/coagulation profile, type and number of blood components requested.

The transfusion data information so obtained from the documents was fed into Microsoft excel sheet for future analysis. The data pertaining to the number of whole blood/packed red cells/fresh frozen plasma/random donor platelet concentrates/single donor apheresis platelet concentrates requested as well as crossmatch/issue data was collected and analyzed.

\section{Result}

During the study period, a total of 2434 (first half $=1210+$ second half=1224) transfusion requisitions were received for 7754 (first half $=2632+$ second half $=2935$ ) blood components. The PRBC requisitions were $3621(49.69 \%)$, $2140 \mathrm{FFP}(27.59 \%), 1923 \mathrm{RDP}$ (24.80\%), whole blood requisitions were $15(0.19 \%)$ and SDP contributed $0.56 \%(55)$ of all transfusion requisitions (Table 1). A total of 5566(71.78\%) blood components were issued where PRBC constituted $41.34 \%$ (2301), FFP as $32.53 \%$ (1811), for platelet concentrates it was $25.08 \%(1396)$, SDP issue was $0.80 \%(45)$ and whole blood was $0.09 \%(13)$. Due to dengue epidemic, there was a seasonal variation in the issue of RDPs (838 vs 558) which led to an increase in the total issued components in the second half of the year 2015. If the variation is ignored, the blood components were issued equally in the both the halves.

Taking first half as representative of the entire year, it was observed that the requisitions from surgical specialities 
$\{62.97 \%(\mathrm{n}=762 / 1210)\}$ exceeded the demand from medical specialities $\{37.02 \%(\mathrm{n}=448 / 1210)\}$. The blood components were utilized for $947(78.26 \%)$ patients amongst 1210 patients requested. Out of total blood components issued, 67.81\% (1784/2631) were transfused to patients for the surgical events and $32.19 \%(847 / 2631)$ to medical ones (Figure 1).

Table I Blood component wise distribution

\begin{tabular}{llllll}
\hline Type of component & PRBC & FFP & RDP & SDP & WB \\
\hline Observations & & & & & \\
Components Requested & 3621 & 2140 & 1923 & 55 & 15 \\
& $49.69 \%$ & $27.59 \%$ & $24.80 \%$ & $0.56 \%$ & $0.19 \%$ \\
Issued & 2301 & 1811 & 1396 & 45 & 13 \\
& $41.34 \%$ & $32.53 \%$ & $25.08 \%$ & $0.80 \%$ & $0.09 \%$
\end{tabular}

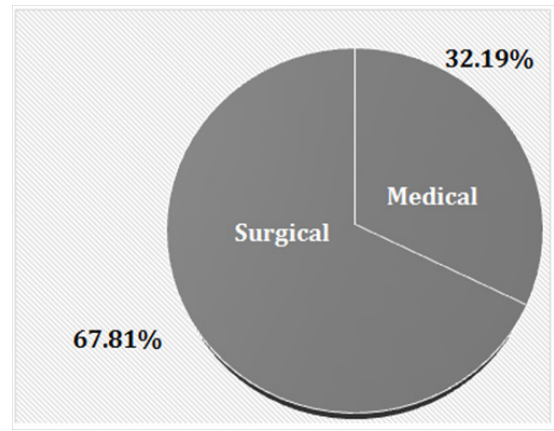

Figure I Specialty wise distribution of issue of blood components.
The age of the admitted patients varied between 3 days to 90 years with male: female ratio being 1.9:1. The maximum PRBC requisitions were received from the patients in the sixth decade of life followed by seventh (Figure 2). The commonest indication for packed red cell transfusion in our hospital was elective surgery ( $\mathrm{N}=577 / 1210,47.7 \%)$ followed by anemia (N=376/1210,31.07\%) and bleeding due to road traffic trauma or postoperative blood loss ( $\mathrm{N}=64 / 1210,5.29 \%)$. B $\mathrm{Rh} \mathrm{D}$ positive (38.01\%) blood components were maximally requested followed by A Rh D positive (25.70\%), O Rh D positive (22.72\%) and $\mathrm{AB}$ Rh D positive $(10.33 \%)$ blood group in the hospital. The mean pre-transfusion hemoglobin trigger for packed red cell transfusion was $7.42 \mathrm{~g} / \mathrm{dl}$. The overall $\mathrm{CT}$ ratio at the hospital was 1.57 and the highest being for CTVS as 1.92 followed by general surgery (1.84) and NSU (1.79). The Transfusion probability and TI were observed as $79.0 \%$ and 1.18 respectively in our study Table 2 .

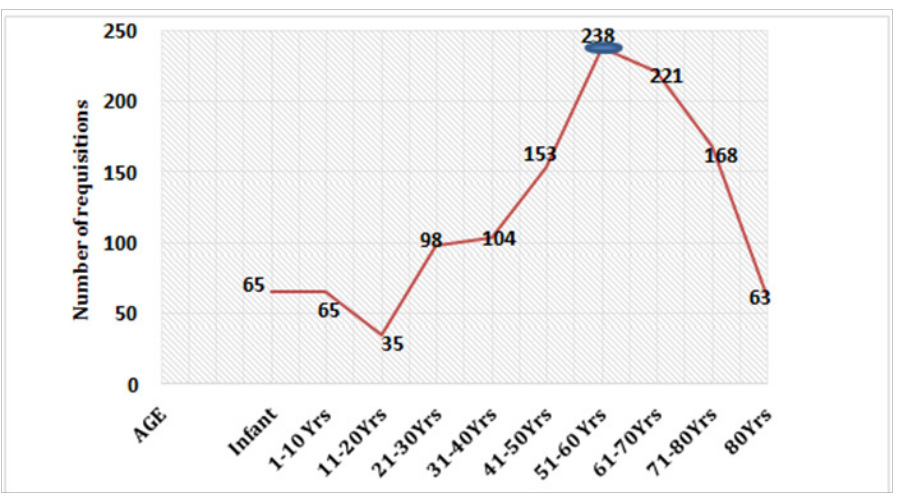

Figure 2 Age distribution of patients.

Table 2 Quality indicators for blood utilization

\begin{tabular}{lllll}
\hline Specialty & CT ratio & Transfusion probability (TP) (\%) & Transfusion index (TI) & MSBOS \\
\hline CTVS & 1.92 & 89.75 & 1.36 & 2.04 \\
General Surgery & 1.84 & 71.42 & 1.3 & 1.95 \\
Neurosurgery & 1.79 & 71.91 & 1.08 & 1.65 \\
Onco surgery & 1.6 & 80 & 0.85 & 1.27 \\
Obstetrics \& Gynecology & 1.43 & 81.08 & 1.3 & 1.95 \\
Pediatric Surgery & 1.42 & 100 & 0.7 & 1.05 \\
Orthopedics & 1.37 & 57.36 & 1.95 & 2.92 \\
Urology & 1.3 & 84.2 & 0.37 & 0.55 \\
Plastic Surgery & 1.28 & 61.53 & 1 & 1.5 \\
Critical Care & 1.46 & 89.5 & 1.75 & 2.62 \\
Cardiology & 1.46 & 80 & 0.87 & 1.3 \\
Nephrology & 1.15 & 92.85 & 1.13 & 1.69 \\
Gastroenterology & 1.13 & 100 & 1.12 & 1.68 \\
Medicine & 1.02 & 97.61 & 1.12 & 1.68 \\
Overall & 1.57 & 79 & 1.18 & - \\
\hline
\end{tabular}

\section{Discussion}

The transfusion of blood or blood components is one of the most significant part of delivery of healthcare services in a hospital setting. ${ }^{1}$ It is quite challenging to maintain a balance between the ongoing blood demand and supply. Appropriate use of blood or blood components is an extremely cost-effective practice but holding blood units for elective surgery and then not transfusing the requested ones add to the testing cost for a patient, fritter away reagent and overburdens a blood bank. There is a great likelihood of low supply because of blood units kept on hold for 48-72hours as per any institutional policy. 
In the present study, the total blood utilization at our centre was observed to be $71.78 \%(\mathrm{n}=5566 / 7754)$ whereas for the surgical specialities alone it was $59.42 \%$. The utilization rate as studied by various centres worldwide for surgical patients varies from 13.6$43.6 \%{ }^{2-7}$ At our hospital, which is a superspecialty one, the maximum requisitions were received from cardiothoracic and vascular surgery (CTVS) unit followed by neurosurgery unit (NSU) and orthopedic unit. CTVS specialty contributed $41.7 \%$ of total blood units crossmatched among all surgical specialities. During the study period, $51.90 \%$ of the total packed red cell units crossmatched for the cardiac patients were transfused whereas the FFP utilization was higher as being $82.20 \%$. It was found that $48.1 \%$ of the blood so crossmatched was unutilized for cardiac surgeries, $44.2 \%$ for NSU and $27.1 \%$ for orthopedic unit among the maximum blood ordering departments which is similar to the observations made by Subramanium et al. ${ }^{8}$

The maximum number of patients transfused were of the age group between 51 to 60years followed by 61 to 70 years while a study from South India and Saudi Arabia observed an average age of $35.12 \pm 20.4$ years. ${ }^{9,10}$ As the majority of the patients requiring blood transfusion in our study were the cardiac patients admitted for elective surgery and hence the age corroborated with the same. The mean hemoglobin observed in our study for all the blood requests was $7.47 \mathrm{~g} / \mathrm{dl}$ which is similar to the observations made by Frank et al. ${ }^{11}$ highlighting that higher number of requisitions for the blood transfusion at our centre were received for surgical $(62.97 \%)$ indications than medical ones $(37.02 \%)$.

A CT ratio of 2.5 or below, Transfusion probability of $\geq 30 \%$ and TI of more than 0.5 is considered indicative of efficient blood utilization as conceptualized by Boral et al. ${ }^{12}$ and Mead et al. ${ }^{13}$ The overall CT ratio at the hospital was 1.57 and the highest being for CTVS as 1.92 followed by general surgery (1.84) and NSU (1.79) suggesting significant blood utilization in contrast to several other studies worldwide and in India, where the CT ratio was very high ranging from 14.16 to $41.4 .^{14-16}$ The key issue remains an over ordering of blood units due to individualistic estimation of anticipatory excessive surgical blood loss and in view of the patient safety. Transfusion probability as low as $4.9 \%$ to $8.8 \%$ and $11.15 \%$ to $47 \%$ have been reported by many authors and the present study shows $79 \%$ indicating appropriate blood utilization. ${ }^{3,6,14-17}$ The TI for the current study was 1.18 which is appropriate as per standards while other authors report $\mathrm{TI}$ as low as $0.06-0.11 .{ }^{14-17}$ Another study by Devi et al. ${ }^{18}$ has reported even better utilization results than ours as CT ratio of 1.02 , TP of $97.2 \%$ and TI of 0.97 highlighting significant blood utililisation and efficient blood transfusion practices at their centre. ${ }^{18}$

Roger et al. ${ }^{19}$ and Vrotsos et al. ${ }^{20}$ observed a significant decline in CT Ratio from 3.21 to1.62 and 2.48 to 1.50 respectively when the later formed a Blood Utilization Committee as the already existing Hospital Transfusion Committee could not bridge the gap between transfusion services and other departments, nursing and clinicians on blood utilization aspect. ${ }^{19,20}$ The highest MSBOS was observed with the Orthopedic unit (2.92) followed by CTVS (2.04), Obstetrics and Gynecology /general surgery (1.95) and neurosurgery unit (1.65) (Table 2). There is a great need to discuss type and screen approach vs crossmatched protocol at our centre which can further lower the resource wastage due to holding of blood units as reported by Jayaranee et al. ${ }^{21}$

There are numerous factors which can affect the blood transfusion practices at a hospital including the blood conservation techniques being used by surgeons, level of training of technologists and nursings, sharing of blood utilization information with other departments. ${ }^{22}$ The encouraging pattern of blood utilization at our centre reflects the positive impact of regular meetings of hospital transfusion committee where all the major blood consumer departments participate and discuss the data and transfusion practices. Any issues related to the turnaround time, availability of units in emergency and holding the crossmatched blood units are discussed and resolved if need there be. However, still there is a big scope of curbing the over ordering of blood units as per each surgical specialty and revise the existing MSBOS for such.

\section{Conclusion}

The study helped in assessment of the transfusion practices at our centre which is found to be encouraging. The CT ratio, transfusion probability and transfusion index demonstrated that there is significant blood utilization at our hospital. But excessive blood ordering for elective surgeries has been observed which leads to wastage of reagental resources, further adding extra cost to the patient. Therefore, the transfusion auditing should be made a common feature with hospital transfusion committee meetings wherein MSBOS can be modified and implemented progressively. Various measures to improve upon the safety and efficiency of blood supply should be put into practice including regular auditing of blood transfusion services at the centre, preparing and following evidence based blood transfusion guidelines and regular training of the clinical residents and the nursing staff.

\section{Acknowledgements}

None.

\section{Conflict of interest}

The author declares no conflict of interest.

\section{References}

1. Teresa J Nel. Clinical guidelines, audits and hemovigilance in managing blood transfusion needs. Transfusion Alternatives in Transfusion Medicine. 2008;10(2):61-69.

2. Basnet RB, Lamichhane D, Sharma VK. A study of blood requisition and transfusion practice in surgery at Bir hospital. PMJN. 2009;9(2):14-19.

3. Vibhute M, Kamath SK, Shetty A. Blood Utilization in elective general surgery cases: requirements, ordering and transfusion practices. $J$ Postgrad Med. 2010;46(1):13-17.

4. Paramjit K, Sabita B, Gagandeep K, et al. An analysis of the pattern of blood requisition and utilization in a tertiary care centre. NJIRM. 2013;4(2):123-127.

5. Mwambungu A, Nathan S, Duncan M, et al. Analysis of blood cross matching ordering practice in surgical patients at Ndola central hospital. International Journal of Healthcare Sciences. 2015;3(1):278-284.

6. Belayneh T, Messele G, Abdissa Z, et al. Blood requisition and utilization practice in surgical patients at university of Gondar hospital, Northwest Ethiopia. J Blood Transfus. 2013;758910.

7. Abubakar UM, Mohammed AN, Abdul AH, et al. Pattern of blood transfusion request and utilization at a Nigerian University Teaching Hospital. Sahel Medical Journal. 2014;17(1):19-22.

8. Subramanian A, Sagar S, Kumar S, et al. Maximum surgical blood ordering schedule in a tertiary trauma center in Northern India: A proposal. J Emerg Trauma Shock. 2012;5(4):321-327. 
9. Apuca Susan M, Surupa Susan K, Nadaraja Pillai S, et al. Pattern of blood component utilization in a teaching hospital in South Kerela. Academic Medical Journal of India. 2014;2(1):28-31.

10. Tayara BK, Faraidy MH, Sayel FA, et al. Blood utilization in orthopedic and trauma practice. Int J Appl Basic Med Res. 2015;5(2):111-113.

11. Frank SM, Savage WJ, Rothschild JA, et al. Variability in blood and blood component utilization as assessed by an anesthesia information management system. Anesthesiology. 2012;117(1):99-106.

12. Boral LI, Henry JB. The type and screen: a safe alternative and supplement in surgical procedures. Transfusion. 1977;17(2):163-168.

13. Mead JH, Anthony CD, Sattler M. Hemotherapy in elective surgery: An incidence report, review of the literature, and alternatives for guideline appraisal. Am J Clin Pathol. 1980;74(2):223-227.

14. Khoshrang H, Madani AH, Roshan ZA, et al. Survey on blood ordering and utilization patterns in elective urological surgery. Blood Transfus. 2012;11(1):123-127.

15. Couture DE, Ellegala DB, Dumont AS, et al. Blood use in cerebro vascular neurosurgery. Stroke. 2002;33(4):994-997.

16. Rehmatullah S, Muhammad RJ, Ahmad AS. Arrangements and use of blood in elective surgical procedures. Professional Medical Journal. 2011;18(2):212-214.
17. Yazdi AP, Alipour M, Jahanbakhsh SS, et al. A survey of blood request versus blood utilization at a university hospital in Iran. Arch Bone Jt Surg. 2015;4(1):75-79.

18. Devi KM, Barindra Sharma A, Dorendro Singh L, et al. Quality indicators of blood utilization in a tertiary care centre in the North-eastern India. IOSR Journal of Dental and Medical Sciences. 2014;13(1):50-52.

19. Rogers BA, Johnstone DJ. Audit on the effective use of crossmatched blood in elective total hip and total knee replacement. Ann R Coll Surg Engl. 2006;88(2):199-201.

20. Vrotsos E, Gonzalez B, Goldszer RC, et al. Improving blood transfusion practice by emphasis of the blood utilization committee :experience of one hospital. Transfus Clin Biol. 2015;22(1):1-4.

21. Jayaranee S, Prathiba R, Vasanthi N, et al. An analysis of blood utilization for elective surgery in a tertiary medical centre in Malaysia. Malays $J$ Pathol. 2002;24(1):59-66.

22. Novis DA, Renner S, Friedberg R, et al. Quality indicators of blood utilization : three College of American Pathologists Q-Probes studies of 12288404 red blood cell units in 1639 hospitals. Arch Pathol Lab Med. 2002;126(2):150-156. 